another pathogenic point mutant. Putting everything together - the calciumdependent calcium channel activity, the localization to the ER and the enhanced calcium release activity - it seems that polycystin-2 may be a new type of calcium release channel, with properties that allow it to mediate calcium-induced calcium release (CICR). Moreover, channel function and the ability to augment calcium release were inhibited in the pathogenic mutants.

The paper will be significant for channel physiologists interested in intracellular calcium signalling, because it proposes that polycystin-2 is a new type of calcium release channel, in addition to ryanodine receptors and $\mathrm{IP}_{3}$ receptors. This paper also resonates with the TRP field. There are about 20 TRP-related genes that can often function as nonselective cation channels, which often exhibit calcium permeability and can be activated in numerous ways. In addition to having a pore region similar to TRP, polycystin-2 can physically associate with TRPC1 (ref. 9). Physical linkage between proteins in the surface membrane and the endoplasmic reticulum may mediate capacitative calcium entry and most certainly underlies excitation-contraction coupling in skeletal muscle. Among the mysteries that remain are how polycystin-2 coupled to polycystin-1 can result in channel activity ${ }^{5}$ and how polycystin-2 channel dysfunction and possible calcium dysregu- lation can cause the disease phenotype found in ADPKD.

Michael D. Cahalan is in the Department of

Physiology and Biophysics, University of California,

Irvine, CA 92697-4561, USA

e-mail:mcahalan@uci.edu

1. Ashcroft, F. in Ion Channels and Disease (Academic Press, 2000),

2. Somlo, S., \& Ehrlich, B. Curr. Biol. 11, R356-360 (2001).

3. Koulen, P. et al. Nature Cell Biol. 4, 191-197 (2002).

4. Gonzalez-Perrett, S. et al. Proc. Natl Acad. Sci. USA 98, 1182-1187 (2001)

5. Hanaoka, K. et al. Nature 408, 990-994 (2000).

6. Vassilev, P. M. et al. Biochem. Biophys. Res. Commun. 282 341-350 (2001).

7. Cai, Y. et al. J. Biol. Chem. 274, 28557-28565 (1999).

8. Tsiokas, L., Kim, E., Arnould, T., Sukhatme, V. P. \& Walz, G. Proc. Natl Acad. Sci. USA 94, 6965-6970 (1997).

9. Tsiokas, L. et al. Proc. Natl Acad. Sci. USA 96, 3934-3939 (1999).

\title{
Getting hit by SUMO
}

Proteins can be targeted to different fates through post-translational modifications. For instance, the addition of ubiquitin (or some other ubiquitin-like proteins) to lysine residues or to the amino terminus of proteins can induce degradation by the proteasome, intracellular trafficking or activation. Targeting by one particular ubiquitin-like protein, the small ubiquitin-related modifier 1 (SUMO1) can affect protein interactions, subcellular localization or stability. Now Frauke Melchior and her colleagues at the Max-Planck Institute in Martinsried, Germany, describe a new link between protein SUMOylation and nuclear transport.

Modification of proteins by ubiquitin is a three-step process that involves three classes of enzymes, E1-E3. The E1 activating enzyme and the E2 conjugating enzyme prime ubiquitin for ligation to a substrate by an E3 ubiquitin ligase. There are several E3 ligases that are responsible for transferring activated ubiquitin to their substrates. These confer substrate specificity. An E1 (Aos1/Uba2) and an E2 (Ubc9) enzyme have been previously described for SUMO1. More recently, RING-domain-containing proteins of the protein inhibitors of activated STATs (PIAS) family have been identified as E3 ligase enzymes for SUMO1. Now, Melchior and colleagues show that the nucleoporin RanBP2, which has been previously characterized for its function in nuclear transport, is also an E3 ligase for SUMO1. They find that purified RanBP2 exerts E3 ligase activity on some SUMO1 targets in vitro (such as Sp100) but not on all of them (indeed p53 could be SUMOylated by PIAS1, but not by RanBP2), indicating substrate specificity. Furthermore, depletion of RanBP2 and its binding partner RanGAP1 from cytosolic extracts results in the loss of SUMOylation activity, which can be rescued by adding back immunoprecipitates containing RanBP2.

Interestingly, the domain of RanBP2 that contains the E3 ligase activity bears no structural, and apparently no functional, homology to either RING finger motifs or HECT domains, two domains that have been previously shown to bear ubiquitin E3 ligase activity in other proteins. This suggests that the mechanism of RanBP2 activity is different to ubiquitin E3 ligases.

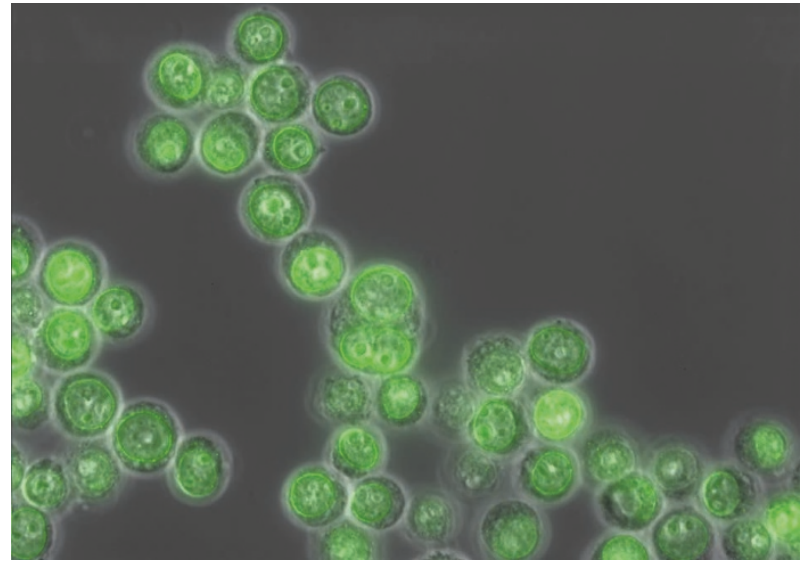

Melchior and coworkers also describe the localization of a fluorescently tagged SUMO1 protein (shown in green in the figure) to the nucleus and to the cytoplasmic side of the nuclear pore complex (NPC). As RanBP2 is part of the machinery that mediates nucleocytoplasmic transport of proteins with a nuclear localization signal (NLS), Melchior's findings provide a rationale for the observation that most SUMO1 target-proteins require an NLS for in vivo modification. Active import seems to contribute to the accumulation of tagged SUMO1 in the nucleus. Furthermore, tagged SUMO1 at the cytoplasmic side of the NPC seems to be conjugated to unknown proteins, consistent with the idea that conjugation of SUMO1 to targets by RanBP2 precedes import. Altogether, these findings raise some interesting questions about the relationship between nuclear transport and SUMOylation. These two events seem to be coordinated, and one can even speculate that SUMOylation could be involved in the translocation process itself.

VALERIE FERRIER 